\title{
A Clustering Algorithm Based on Zone in Vehicular Ad Hoc Networks
}

\author{
* Zhijun Yan ${ }^{1}$, Yang Tao ${ }^{2}$, Fangjin Zhao ${ }^{3}$ and QianJin $\mathrm{He}^{4}$ \\ Institute of Communication and Information Engineering, The ChongQing \\ University of Posts and Telecommunication, ChongQing, China \\ I'goforbeauty@163.com, ${ }^{2} 928351320 @ q q . c o m$
}

\begin{abstract}
Vehicular Ad Hoc Networks (VANET) is a wireless mobile ad hoc networks established on the inter-vehicle communication. Due to the special characteristics and the restrictions of roads, VANET shows characteristics of uneven nodes density, fast moving, high dynamic topology. So it is difficult to establish a stable link between nodes, and the reliability of data transmission declines rapidly with the increase of hops. Therefore, ensuring data reliability and rapid distribution is always a difficult point in the VANET research. Through analysis of vehicular ad hoc networks environment, we propose the node connectivity and the connectivity strength. By dividing the road environment into the segment area and the intersection area, respectively calculating and predicting the connectivity according to the road environment, we propose the zone based adaptive clustering algorithm (ZACA).
\end{abstract}

Keywords: clustering algorithm, node connectivity, connectivity strength, zone

\section{Introduction}

As the original research part of Intelligent Transportation system (ITS [1]), the communication between vehicles is the key research of VANET [2-3]. R. Baskaran [4] proposes a bio-inspired artificial bee colony approach to overcome the connectivity and the signal fading issue. Mohamed Aissa [5] proposes a scalable weighted clustering algorithm by implementing a non-periodic procedure for clusterhead election. This algorithm can increase the cluster formation and stability. But the connectivity is not considered. Ambuj Kumar and other authors [6] put forward a cluster strategy based on vehicles' direction correlation degree. The strategy divides vehicles into different groups according to the relative velocity, and neighboring vehicles with similar velocity are the same group. It also selects a group leader (GL) for broadcast packet forwarding and improves the stability and survival time of clusters. But the way of clustering based on relative velocity also restricts its application range and could not apply to city roads or roads in a complex environment.

Through analysis of the traditional clustering algorithms [7-8], most of them take the node connectivity less into consideration. Even considering the node connectivity, they concern less about the node connectivity strength and prediction. So, based on characteristics of VANETS which is uneven node density, high dynamic network topology and complex wireless environment, we put forward the node connectivity strength and the node connectivity prediction. Then, the zone based adaptive clustering algorithm (ZACA) is proposed. Through dividing the road environment into the segment area and the intersection area, transfer the management of massive uncertainty topology structure within in-vehicle network into more effective management within the local cluster. Given that, the clustering stability is improved efficiently under in-vehicle network. 


\section{The Zone Based Adaptive Clustering Algorithm}

\subsection{The Analysis of the Connectivity in Area Network}

In order to describe the network connectivity[9] in VANET, add factors of the node degree, the node velocity, the moving direction and the velocity of neighboring nodes at the $t$ moment for measuring network connectivity. And we also introduce the node connectivity, the node connectivity strength, and the regional connectivity. Definition as follows:

VANET network topology can be regarded as an undirected graph which is constituted by nodes and links and denoted by $G(V, E) . V$ is the set of vehicle nodes and $E$ is the edge set of communication links. In this undirected graph, vehicle nodes can be regarded as points and communication links between two nodes can be regarded as edges.

The node connectivity $N_{a}(t)$ is the sum of neighbor nodes which can be connected around the node $a$ within some time period $t . N_{a}(t)$ can measure connectivity status of the node $a$ within some time period $t$, as follows:

$$
N_{a}(t)=\sum_{i=0}^{m} i\left\{i \mid D_{a i}(t) \leq D_{t h}\right\}
$$

$D_{a i}(t)$ is the distance between the node $a$ and the node $i$ within some time period $t$ and $D_{t h}$ represents the distance threshold and its value is set by the network, $D_{t h} \leq r$, where $r$ is vehicle communication radius.

Node connectivity strength $Q_{a}(t)$ is the value of average time-period of link connected between node $a$ and node $i$ within some time period $t$, as the formula:

$$
Q_{a}(t)=\left(\sum_{i=0}^{m} T_{a i}(t)\right) / m
$$

In this formula, $T_{a i}(t)$ represents the time of the connected link which the node $a$ and the node $i$ predict and keep within some time period t. $T_{a i}(t)$ has different prediction algorithm under different road conditions. $m$ represents the node connectivity $N_{a}(t)$ at current time.

The regional connectivity $C_{z}(t)$ represents the node average connectivity within the network area $\mathrm{Z}$ and effectively characterize the nodes' area network connectivity, as the formula of $C_{z}(t)$ follows:

$$
C_{z}(t)=\left(\sum_{i=0}^{n} N_{i}(t)\right) / n
$$

In this formula, $i$ represents nodes within the network area $\mathrm{Z}$ and $N_{i}(t)$ represents the connectivity of the node $i$ while $n$ represents the number of nodes. Since the area $\mathrm{Z}$ composed by the segment area and the intersection area, $\mathrm{Z}$ is related with vehicular network environments.

\subsection{Node Connectivity Prediction}

According to characteristics of urban road environment, divide roads into the section model (Segment Model) and the intersection model (Intersection Model) and 
compute the connectivity time of nodes' link for nodes' connectivity prediction [10] in VANET.

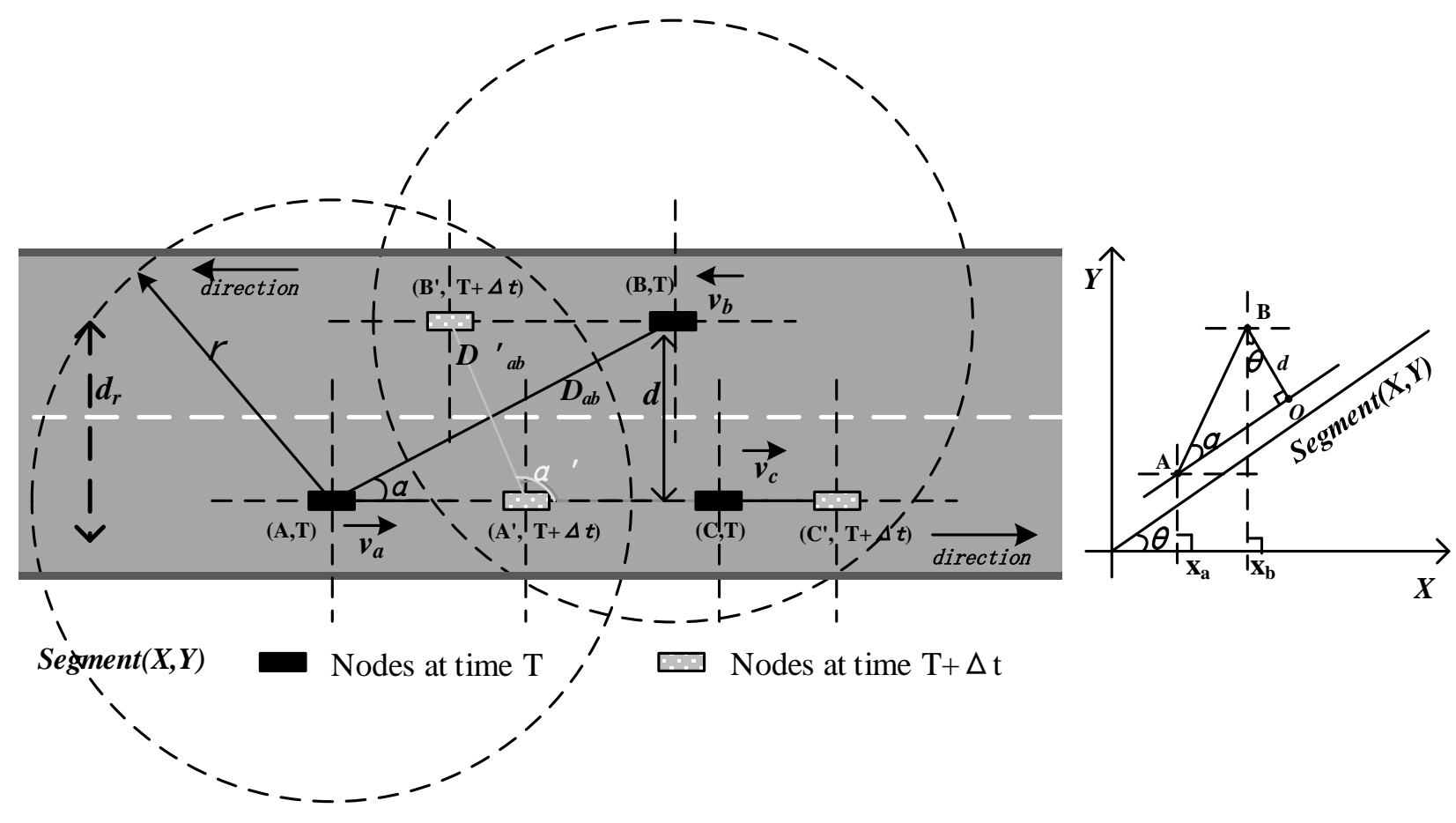

Figure 1. Segment Connectivity Prediction

In the road model, vehicles' position relationships can be approximately in a straight line and vehicles' movement is divided into the same direction and the opposite direction. When $\mathrm{A}$ and $\mathrm{B}$ are connected at time $t$, the connectivity time $T_{a b}(t)$ satisfies the formula:

$$
D_{a b}\left(t+\mathrm{T}_{a b}(t)\right) \leq D_{t h}
$$

From Figure 1 and further analysis, the formula of $T_{a b}(t)$ as follows:

$$
T_{a b}(t)=\frac{\sqrt{D_{t h}^{2}-d^{2}} \llbracket \cos \theta+\left|x_{a}(t)-x_{b}(t)\right|+d \llbracket \sin \theta}{\Delta v_{a b} \llbracket \cos \theta}
$$




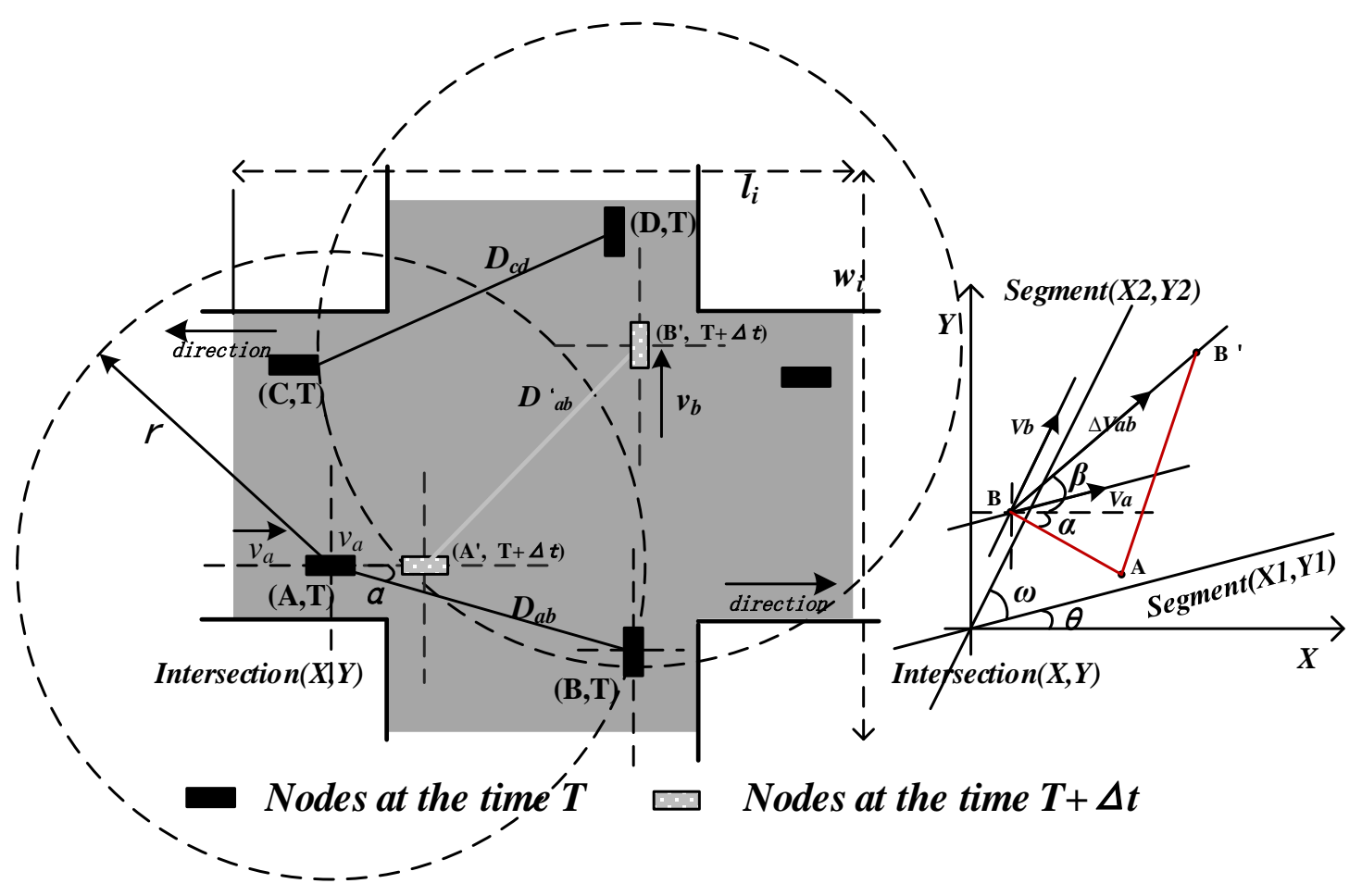

Figure 2. Intersection Connectivity Prediction

From the Figure 2, when the vehicle A and B both are in the Intersection (x,y) and vehicles leave the road to enter the different segments, $T_{a b}(t)=0$. If $\mathrm{A}$ and $\mathrm{B}$ moving directions are not in the same segment and the distance is less than or equal to $D_{t h}$, link connected time $T_{a b}(t)$ can be described as follows:

$$
T_{a b}(t)=\frac{-\left(\Delta v_{x} \llbracket \Delta x+\Delta v_{y} \square \Delta y\right)+\sqrt{\left[\left(\Delta v_{x}\right)^{2}+\left(\Delta v_{y}\right)^{2}\right] D_{t h}{ }^{2}-\left(\Delta v_{x} \square \Delta v_{y}-\Delta x \llbracket \Delta\right)^{2}}}{\left(\Delta v_{x}\right)^{2}+\left(\Delta v_{y}\right)^{2}}
$$

$\Delta v_{x}$ and $\Delta v_{y}$ respectively represent different values of the horizontal component and the vertical component of $\mathrm{A}$ and $\mathrm{B}$ 's velocity at $\Delta t . \Delta x$ and $\Delta y$ respectively represent difference values of the horizontal component and the vertical component of $\mathrm{A}$ and B's velocity at the time $t$.

\subsection{The Zone based on Clustering Algorithm}

Assume that all vehicles with same node transmission radius use omni-directional antenna and all communications are bi-directional links in VANET. Through periodic Beacon Message, Nodes and neighboring nodes gets the information of neighboring nodes.

First, dynamic clustering should be initialized. When initializing cluster begins, all nodes are common nodes $(\mathrm{CN})$ and updates its neighbor table through access to beacon messages of neighboring nodes. Then nodes create or join the cluster and recommend the cluster head node. The clustering initialization process is shown as follows: 


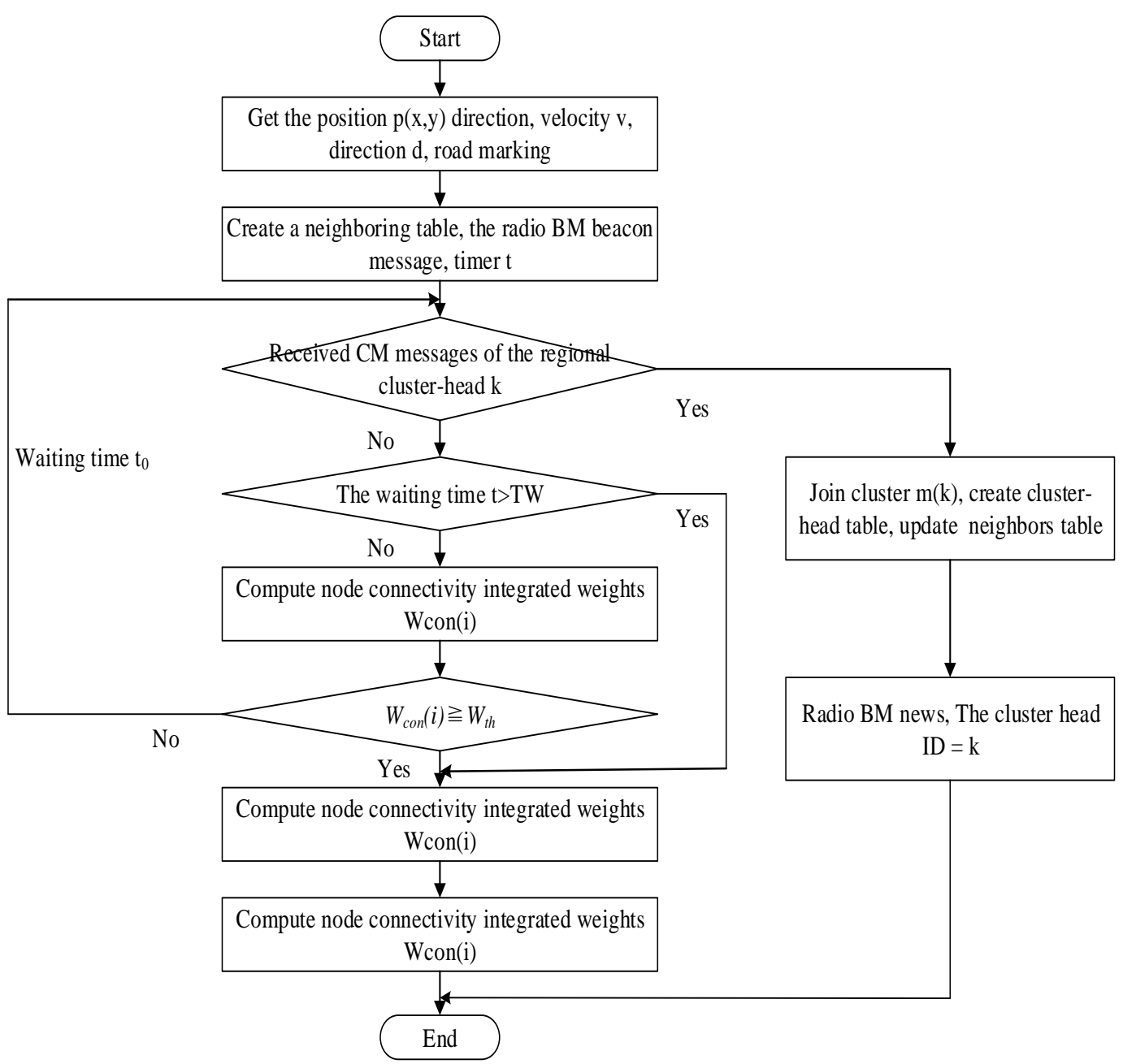

Figure 3. Initializing Clustering Algorithm

For better election of the cluster head, choose the improvement scheme based on WCA [11]. The node with maximum comprehensive weight value has best performance and priority selection for the cluster head node. The maximum comprehensive weight value can be described as follows:

$$
W_{c o n}(i)=\omega_{1} \square \Delta q(i)+\omega_{2} \square \frac{N_{i}}{N_{t h}}+\omega_{3} \square \Delta v(i)+\omega_{4} \square \frac{N_{c}}{N}
$$

Where $\Delta q(i)$ represents the normalized value of the connectivity strength of the node $i$ and shows relative size of the connectivity strength of current and neighbor nodes; The second component $\frac{N_{i}}{N_{t h}}$ represents the connectivity of the current node $i$. The higher it is, the better the connectivity. $N_{i}$ represents the connectivity degree and $N_{t h}$ represents the connectivity degree threshold of the system. The third component $\Delta v(i)$ which satisfies $\Delta v(i) \leq 1$ represents the motion state of the current node $i$. The forth component $\frac{N_{c}}{N}$ represents the direction correlation of current and neighbor nodes. $N$ represents the number of neighbor nodes of $i . N_{c}$ represents the number of neighbor nodes whose movement direction is consistent with node $i . \omega_{1}$, 
$\omega_{2}, \omega 3$ and $\omega 4$ are the weighing factors for the corresponding system parameters. The sum of them equals to 1 .

Vehicle nodes' joining and leaving, the gravity of the cluster head and the cluster merging and splitting are the key point of clustering maintenance. Through the clustering adaptively divided by region, the stability of clusters is enhanced and the network overhead is also reduced. The process is just as followed:

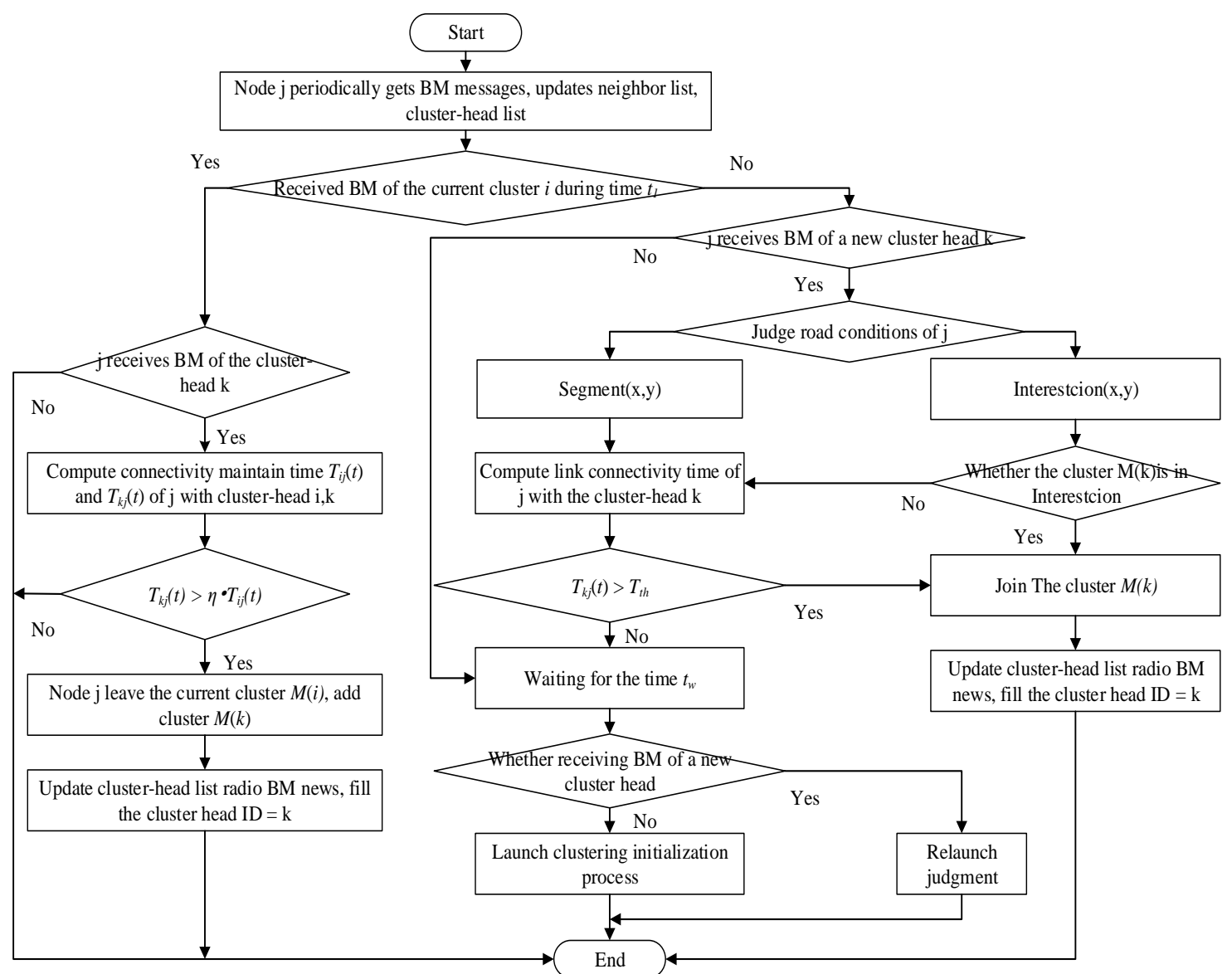

Figure 4. The Maintenance of Nodes' Joining and Leaving

Vehicle nodes are decided by the segment and intersection environment under urban environments. So the distribution of nodes has uneven characteristics. nodes' movement can cause the coverage of clustering communication radius, in order to reduce the cluster stack and reducing cluster redundancy, the clustering scale should be adjusted just as follows: 


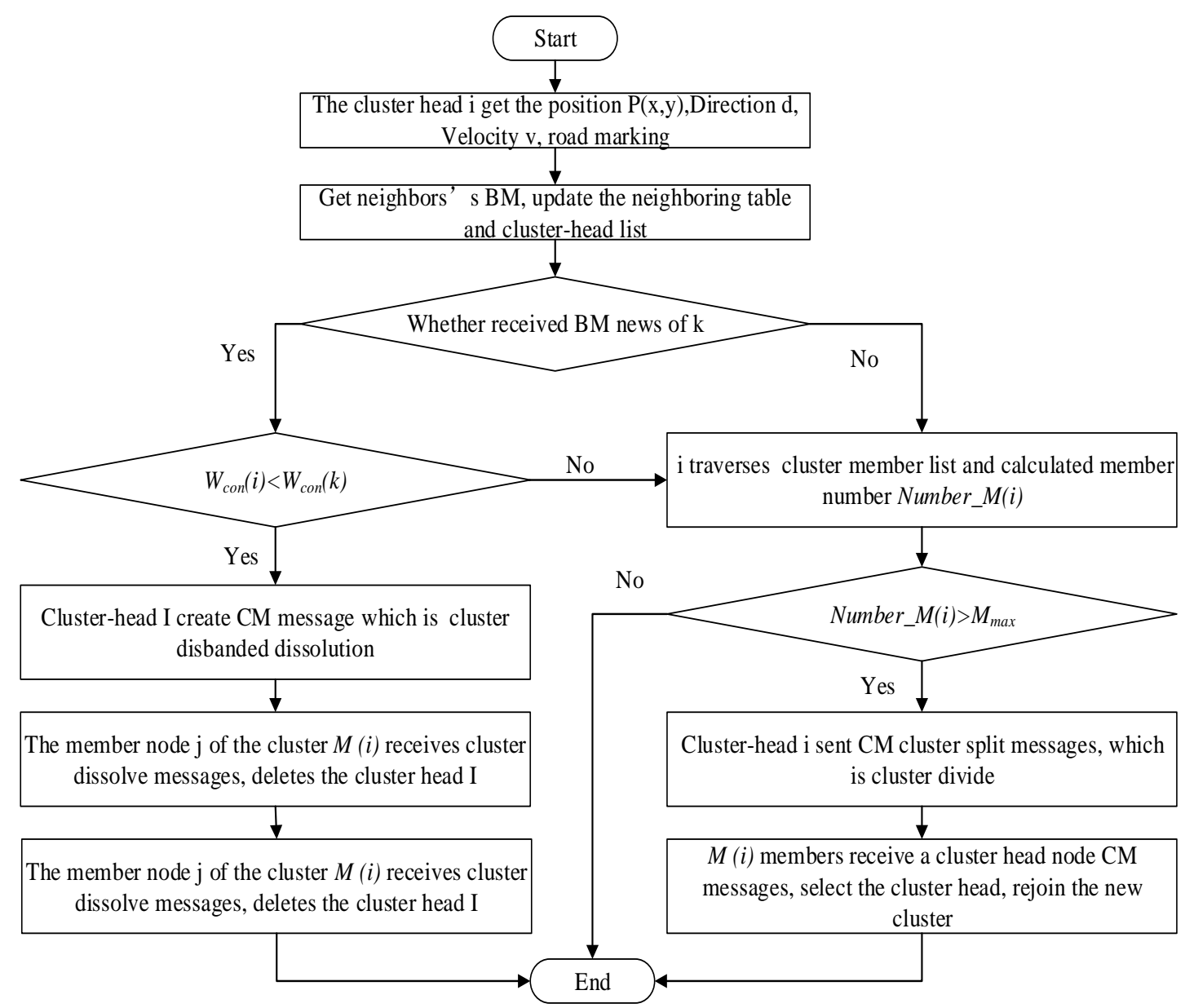

Figure 5. The Maintenance of Nodes' Joining and Leaving

\section{Simulation Analysis}

We use VanetMobiSim Simulator and NS2 for simulation and analysis. Set the simulation area $1900 \mathrm{~m} \times 1200 \mathrm{~m}$ in size and the number of vehicles $[50,100,150,200,250,300]$. Vehicle speed has random value $[0-25] \mathrm{m} / \mathrm{s}$ and the node mobility model uses extension model based on intelligent lane model IDM_IC. The waiting time of nodes in the segment is set random value [0-20] s. The initial position of the node is set a random distribution and the system run time of simulation is $600 \mathrm{~s}$. Under the segment environment, $\omega_{1}, \omega_{2}, \omega 3, \omega 4$ is respectively $0.24,0.26,0.16,0.34$ while under the intersection environment, $\omega_{1}, \omega_{2}, \omega 3, \omega 4$ is respectively $0.26,0.32,0.30,0.12$.Finally, the cluster-head switch parameter $\eta$ is 2.5 , the maximum threshold value of the number of cluster membership is 15 and the threshold value of nodes' distance $D_{\text {th }}$ is 135 .

(1) Cluster-head numbers 


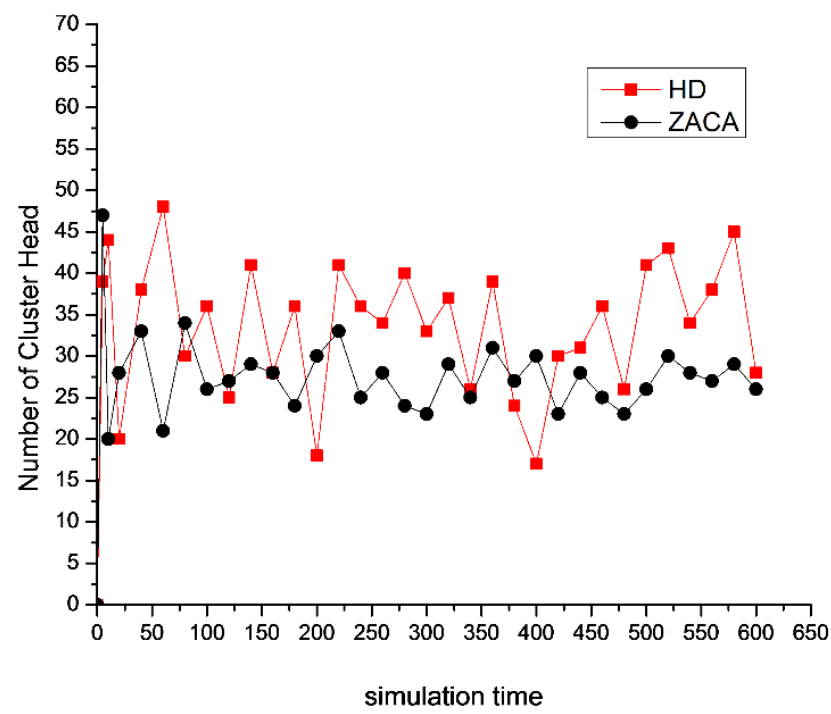

Figure 6. Cluster-head Numbers

We can learn from the simulation results that along with the over simulation time, the number of cluster heads shows the trend of dynamic change. And with the vehicles' moving, the cluster-head number of the ZACA algorithm keeps in the range 22-31 while the cluster-head number of the HD algorithm shows a sharp swing and its average number is also higher than the ZACA algorithm's. The results also prove that through calculations of regional connectivity of nodes in the ZACA algorithm, the cluster-head selection takes fully into account the location and relative stability with neighboring nodes, thus reducing effects of vehicles' movement on clustering. However, in the HD algorithm, the election of the clusterhead node is only judged by nodes with largest numbers of neighboring nodes. Hence the number of cluster-head is larger when the distribution of vehicles is uneven.

(2) The average transfer times per unit time

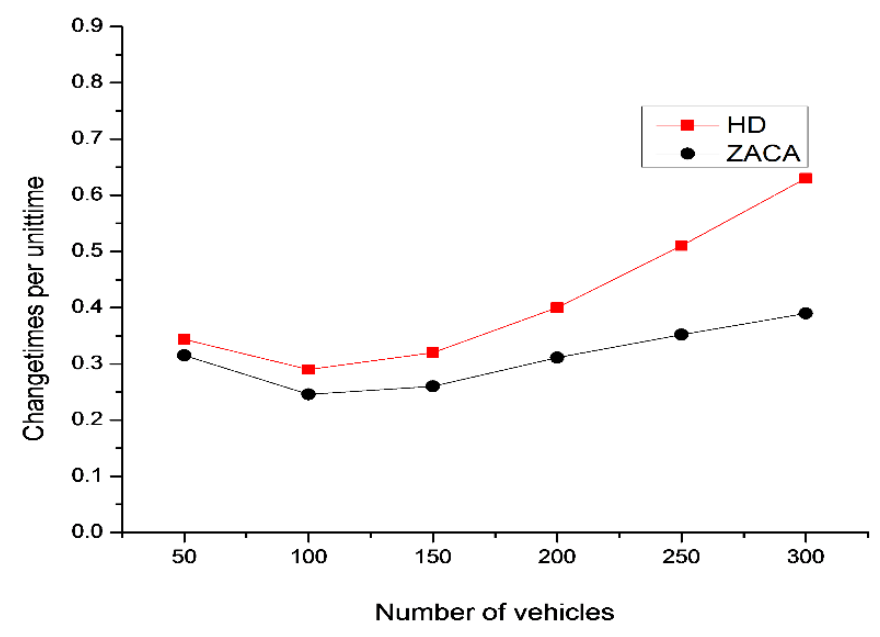

Figure 7. The Average Transfer Times per unit Time 
The figure 6 shows that as the increasing number of vehicles, for both the HD and the ZACA algorithm, the average transfer times per unit time decrease first and then increase. However, the average transfer times per unit time of the ZACA is less than the HD's. From the analysis of simulation results, when the number of network nodes is 50, sparse network nodes cause less number of neighbor nodes within the transmission radius. For the HD algorithm, as vehicles' moving, the great change of the neighboring nodes' number results in more times of algorithm execution. Hence, the node transfer times become more. But, with the increasing number of vehicles, node's average transfer times can keep a lower growth rate for ZACA algorithm and average transfer times is less than 0.35 per unit time. During the interval $[100,150,200,250,300]$, the average times is just $81.2 \%, 84 \%, 69 \%$ and $61.5 \%$ of the average times of the HD algorithm. That is because as the increasing density of vehicles, ZACA can maintain stable network clustering numbers and nodes' transfer between clusters can maintain a stable level.

(3) Update times of cluster-head control sets per unit time

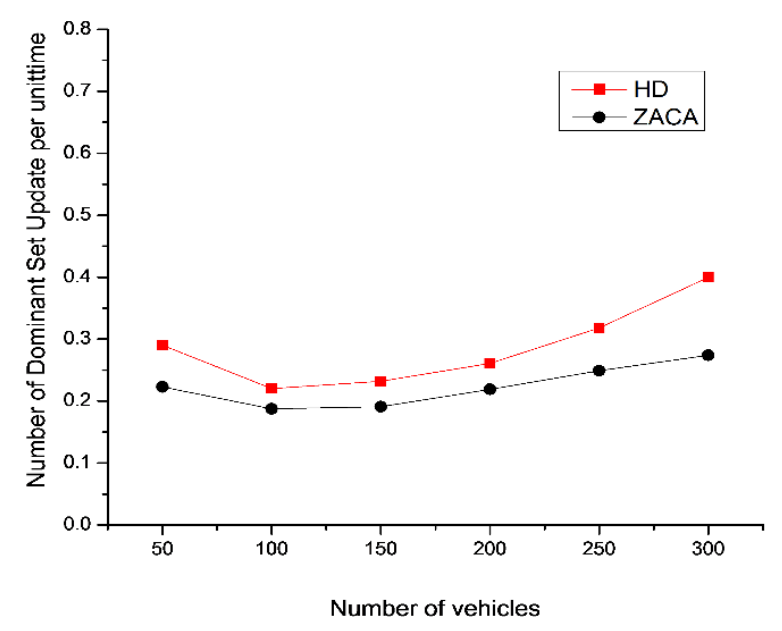

\section{Figure 8. Update Times of Cluster-head Control Sets per unit Time}

Simulation results show that as the increasing number of network nodes, update times of clustering sets of ZACA per unit time are less than HD's and the increasing speed is flat. That also proves that ZACA with high scalability can adapt to the environment of urban roads better and effectively reduce times of clustering algorithms implementation. That is because the ZACA algorithm divides network area into the segment model and intersection model. Therefore, each intersection or the clustering number of intersections are relatively fixed and only when clusterhead nodes leave the current segment and intersection or the current cluster-head nodes overlaps with the neighboring cluster-head nodes are the cluster split and dissolution implemented. When member nodes of clusters find no a cluster head, the clustering algorithm is implemented. Hence, update times of cluster-head control sets caused by vehicles' directions and election of roads and the overhead of clustering can be efficiently reduced 


\section{Conclusions}

In this paper, through deep analysis of VANET and network application environment, we propose the ZACA algorithm. And the simulation results show that ZACA can better adapt to the urban environment and the network has high stability.

\section{References}

[1] W. Fei-Yue, "A Review of IEEE T-ITS: The 2013 Survey Result”, IEEE Journals \& Magazines, vol. 14, no. 2, (2013), pp. 501-510.

[2] S. K. Bhoi, P. M. Khilar and E. F. Nakamura, "Vehicular communication: a survey", IET Networks, vol. 3, no. 3, (2014), pp. 204-217.

[3] M. C. Surugiu and R. V. Alexandrescu, "Analysis of the development and implementation of VANET network intervehiculary communication systems", Electronics, Computers and Artificial Intelligence (ECAI), 2013 International Conference on, Pitest, (2013).

[4] R. Baskran, M. S. S. Basha, J. Amudhavel, K. P. Kumar, D. A. Kumar and V. Vijayakumar, "A bioinspired artificial bee colony approach for dynamic independent connectivity patterns in VANET", Circuit, Power and Computing Technologies (ICCPCT), 2015 International Conference on, Nagercoil, (2015).

[5] M. Aissa and A. Belghith, "A bio-inspired artificial bee colony approach for dynamic independent connectivity patterns in VANET", Information Technology and e-Services (ICITeS), 2013 3rd International Conference on, Sousse, (2013).

[6] A. Kumar and R. P. Nayak, "An efficient group-based safety message transmission protocol for VANET", Communications and Signal Processing (ICCSP), 2013 International Conference on IEEE, (2013), pp. 270-274.

[7] R. Chai, B. Yang, L. Li, X. Sun and Q. Chen, "Clustering-based data transmission algorithms for VANET", Wireless Communications \& Signal Processing (WCSP), 2013 International Conference on,Hangzhou, (2013).

[8] D. Tian, Y. Wang, H. Xia and F. Cai, "Clustering multi-hop information dissemination method in vehicular ad hoc networks", IET Intelligent Transport Systems, vol. 7, no. 4, (2013), pp. $464-472$.

[9] Y. Wei-dong, F. Lin-lin, L. Ji-zhao, "Network connectivity characteristics for vehicular ad hoc network", Journal on Communications, vol. 33, no. 21, (2012), pp. 48-52.

[10] I. W. H. Ho, K. K. Leung and J. W. Polak, "Connectivity dynamics for vehicular ad-hoc networks in signalized road systems", Teletraffic Congress, IEEE, (2009), pp. 1-8.

[11] C. Genle and F. Xiumei, "Research on Cluster-based Trust Routing Protocol in Vehicle Ad Hoc Network", Journal of Tianjin University of Science \& Technology, vol. 28, no. 4, (2013), pp. 70-73.

\section{Authors}
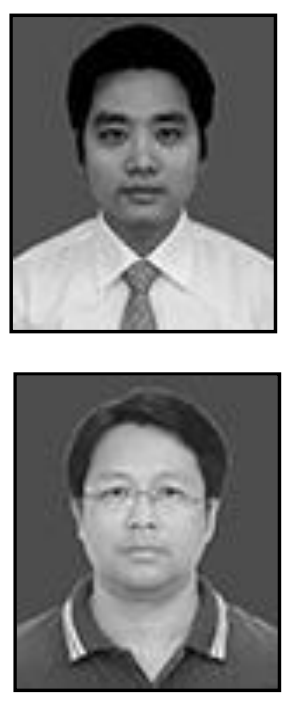

Zhijun Yan, he was born in 1991, is now a master candidate of the college of communication and information engineering, in the ChongQing University of Posts and Telecommunication, China. His main research includes the network application management, the theory of communication networks.

Yang Tao, he was born in 1964, he was received the $\mathrm{PhD}$ degree in Chongqing University China. And $\mathrm{He}$ was received the Postdoctoral degree in the Institute of Computing Technology, Chinese Academy. Now, He working as a Professor in the college of communication and information engineering, in the Chongqing University of Posts and Telecommunication. His main research includes the theory of communication networks, network application management. 


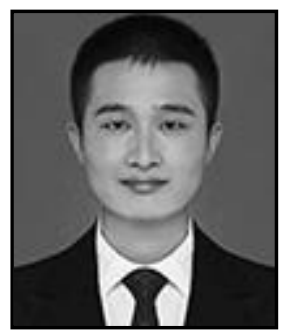

Fangjin Zhao, he was born 1990, is now a master candidate of the college of communication and information engineering, in the ChongQing University of Posts and Telecommunication, China. And his research includes network application management.

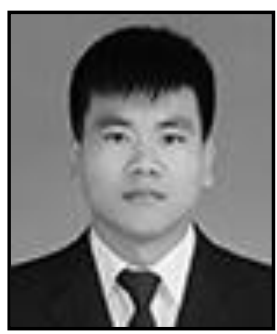

Qianjin He, he was born 1990, is now a master candidate of the colleage college of communication and information engineering, in the ChongQing University of Posts and Telecommunication, China. And his main research is computer network and communication. 
International Journal of Future Generation Communication and Networking Vol. 9, No. 12 (2016) 